\title{
Case Studies in Complementarity: Improving Model Formulation *
}

\author{
Michael C. Ferris ${ }^{\dagger} \quad$ Todd S. Munson ${ }^{\dagger}$
}

November 9, 1998

\begin{abstract}
Over the past several years, many practitioners have been formulating nonlinear variational inequalities as mixed complementarity problems within modeling languages such as GAMS and AMPL. Sometimes the models generated are poorly specified, either because the function is undefined near the solution or the problem is ill-conditioned or singular. In this paper, we look at information provided by the PATH solver about the model that can be used to identify problem areas and improve formulation. Descriptions and uses of the data provided are detailed via several case studies.
\end{abstract}

\section{Introduction}

Developing a practical model of a complex situation is a difficult task in which an approximate representation is initially constructed and then iteratively refined until an accurate formulation is obtained. During the intermediate stages, the models generated have a tendency to be ill-defined, ill-conditioned, and/or singular. Information generated by a solver can help the modeler to detect these problems, quickly locate the source, and make appropriate modifications to the model. By preventing the propagation of errors to successive models, the development cycle shortens and the final product becomes easier to solve with a more meaningful solution. In this paper, we consider equilibrium problems built using the mixed complementarity framework and discuss information provided by the PATH solver that can be used to find potential difficulties. Descriptions and uses of the data provided are detailed via several case studies.

We begin by introducing the mixed complementarity problem (MCP) and briefly review pertinent information about modeling languages and the PATH solver in Section 2. We also discuss merit functions used by solvers to indicate

\footnotetext{
* This material is based on research supported in part by National Science Foundation Grant CCR-9619765 and Air Force Office of Scientific Research Grant F49620-98-1-0417.

${ }^{\dagger}$ Computer Sciences Department, University of Wisconsin, 1210 West Dayton Street, Madison, WI 53706 (ferris,tmunson@cs.wisc.edu)
} 
if an iterate is close to the solution set of the given MCP. We utilize the merit function information in Section 3 when we present the first of our problem areas, ill-defined models. Section 4 addresses poorly-scaled models and the difficulties encountered in such circumstances. Information provided by the solver to detect scaling problems are mentioned. Section 5 concludes our trilogy of problems areas by looking at singular models. Finally, we present a detailed case study in Section 6 on the Von Thunen Land Use model, a classic and difficult problem from the economics literature. The traditional formulation is coded and analyzed using the tools provided by PATH. We conclude that the model developed is poor and use problem specific expertise to improve it.

We remark that the output generated by PATH is not a replacement for the modeler's knowledge about the application, rather it augments the available information to help them rapidly identify potential problem areas.

\section{$2 \quad \mathrm{MCP}$ and Merit Functions}

The mixed complementarity problem is defined by a function, $F: D \rightarrow \mathbf{R}^{n}$ where $D \subseteq \mathbf{R}^{n}$ is the domain of $F$, and possibly infinite lower and upper bounds, $\ell$ and $u$. Let $C:=\left\{x \in \mathbf{R}^{n} \mid \ell \leq x \leq u\right\}$, a Cartesian product of closed (possibly infinite) intervals. The problem is given as

$$
M C P \text { : find } x \in C \cap D \text { s.t. }\langle F(x), y-x\rangle \geq 0, \forall y \in C .
$$

This formulation is a special case of the variational inequality problem defined by $F$ and a (nonempty, closed, convex) set $C$. Special choices of $\ell$ and $u$ lead to the familiar cases of a system of nonlinear equations

$$
F(x)=0
$$

(generated by $\ell \equiv-\infty, u \equiv+\infty$ ) and the nonlinear complementarity problem

$$
0 \leq x \perp F(x) \geq 0
$$

(generated using $\ell \equiv 0, u \equiv+\infty$ ). The notation $\perp$ is used throughout this paper to denote the fact that the enclosing quantities (in this case $x$ and $F(x)$ ) are complementary, that is $\langle x, F(x)\rangle=0$.

Complementarity problems can be easily written within modeling languages such as AMPL[13] and GAMS[1]. Modeling languages offer an environment tailored to expressing mathematical constructs. They can efficiently manage a large volume of data and allow users to concentrate on the model rather than the solution methodology. Details of the modeling language extensions and syntax for complementarity problems can be found in $[6]$ and $[14,9]$. From an algorithmic perspective, modeling languages provide a clean interface to the problem by transforming a general model into a canonical form and providing the solver with access to first and possibly second order derivatives via automatic differentiation. 
A mathematically trivial, but useful modeling feature is when $\ell_{i}=u_{i}$; that is $x_{i}$ is fixed at a finite value. Due to the product nature of $C$ it is easy to see that $F_{i}(x)$ is then unrestricted at all solutions and therefore the size of the MCP can be reduced by fixing the variable $x_{i}$ and removing the function $F_{i}$. Both AMPL and GAMS perform this operation automatically. For the rest of this paper we will assume that all fixed variables have been removed and $\ell<u$.

The PATH solver $[3,4]$ is a damped Newton method based upon the "normal map" operator

$$
F(\pi(x))+x-\pi(x)=0
$$

where $\pi(x)$ represents projection onto the set $C$. The "Newton point" is a solution of the linearization:

$$
L F(\pi(x))+x-\pi(x)=0 .
$$

Several algorithmic enhancements have been made to improve the solver's robustness including non-monotone searching [8], a watchdog strategy, a crash technique [5] to rapidly identify the active set, restarts and artificial variables [10], and the ability to change merit functions and take gradient steps [7]. The current version of the code is highly successful at solving the mixed complementarity problems in the GAMSLIB and MCPLIB [2] test suites. Examples of applications that use the complementarity framework are given in [11, 12].

A solver for complementarity problems typically employs a merit function to indicate the closeness of the current iterate to the solution set. The merit function is zero at a solution to the original problem and strictly positive otherwise. Numerically, an algorithm terminates when the merit function is approximately equal to zero, thus possibly introducing spurious "solutions".

The modeler needs to be able to determine with some reasonable degree of accuracy whether the algorithm terminated at solution or if it simply obtained a point satisfying the desired tolerances that is not close to the solution set. For complementarity problems, we can provide several indicators with different characteristics to help make such a determination. If one of the indicators is not close to zero, then there is some evidence that the algorithm has not found a solution. We note that if all of the indicators are close to zero, we are reasonably sure we have found a solution. However, the modeler has the final responsibility to evaluate the "solution" and check that it makes sense for their application.

For the NCP, a standard merit function is

$$
\left\|(-x)_{+},(-F(x))_{+},\left\langle x_{+},(F(x))_{+}\right\rangle\right\|
$$

with the first two terms measuring the infeasibility of the current point and the last term indicating the complementarity error. In this expression, we use $(\cdot)_{+}$ to represent the Euclidean projection of $x$ onto the nonnegative orthant, that is $(x)_{+}=\max (x, 0)$. For the more general MCP, we can define a similar function, however, we need to make special considerations when we have infinite values in the lower or upper bounds. We define an auxiliary function, $G$, that allows 
us to deal with this situation component-wise as follows:

$$
G_{i}(x, a):=\left\{\begin{array}{cl}
1 & \text { if } a_{i}=\infty \\
x_{i}-a_{i} & \text { if } x_{i} \geq a_{i} \\
0 & \text { otherwise }
\end{array}\right.
$$

Then a corresponding complementarity error is given as

$$
\left\|x-\pi(x),\left\langle G(x, \ell),(F(x))_{+}\right\rangle,\left\langle G(-x,-u),(-F(x))_{+}\right\rangle\right\|
$$

where $\pi(x)$ represents the Euclidean projection of $x$ onto $C$. We can see that if we have an $\mathrm{NCP}$, the function is exactly the one previously given and for nonlinear systems of equations, this becomes $\|F(x)\|$.

There are several reformulations of the MCP as systems of nonlinear (nonsmooth) equations for which the corresponding residual is a natural merit function. Some of these are as follows:

- Generalized Minimum Map: $x-\pi(x-F(x))$

- Normal Map: $F(\pi(x))+x-\pi(x)$

- Fischer Function: $\Phi(x)$, where $\Phi_{i}(x):=\phi\left(x_{i}, F_{i}(x)\right)$ with

$$
\phi(a, b):=\sqrt{a+b}-a-b .
$$

Note that $\phi(a, b)=0$ if and only if $0 \leq a \perp b \geq 0$. A straightforward extension of $\Phi$ to the MCP format is given for example in [7].

In the context of nonlinear complementarity problems the generalized minimum map corresponds to the classic minimum map $\min (x, F(x))$. Furthermore, for NCPs the minimum map and the Fischer function are both local error bounds and were shown to be equivalent in [15]. Figure 3 in the subsequent section plots all of these merit functions for the ill-defined example discussed therein and highlights the differences between them.

The squared norm of $\Phi$, namely $\Psi(x):=\frac{1}{2} \sum \phi\left(x_{i}, F_{i}\right)^{2}$, is continuously differentiable on $\mathbf{R}^{n}$ provided $F$ itself is. Therefore, the first order optimality conditions for the unconstrained minimization of $\Psi(x)$, namely $\nabla \Psi(x)=0$ give another indication as to whether the point under consideration is a solution of MCP.

The merit functions and the information PATH provides at the solution can be useful for diagnostic purposes. By default PATH 4.0 returns the best point with respect to the merit function because this iterate likely provides better information to the modeler. As detailed in [7], the default merit function in PATH 4.0 is the Fischer function. To change this behaviour the merit_function option can be used. Other added options to control the output discussed in this paper are described in Table 1. 


\begin{tabular}{|c|c|c|}
\hline Option & Default & Explanation \\
\hline output_factorization_singularities & no & $\begin{array}{l}\text { Report on the linearly dependent } \\
\text { columns encountered during fac- } \\
\text { torization. }\end{array}$ \\
\hline output_final_degeneracy_statistics & no & $\begin{array}{l}\text { Print information regarding de- } \\
\text { generacy at the solution. }\end{array}$ \\
\hline output_final_point_statistics & yes & $\begin{array}{l}\text { Output information about the } \\
\text { point, function, and Jacobian at } \\
\text { the final point. }\end{array}$ \\
\hline output_final_scaling_statistics & no & $\begin{array}{l}\text { Display matrix norms on the Ja- } \\
\text { cobian at the final point. }\end{array}$ \\
\hline output_final_statistics & yes & $\begin{array}{l}\text { Output evaluatation of available } \\
\text { merit functions at the final point. }\end{array}$ \\
\hline output_initial_point_statistics & yes & $\begin{array}{l}\text { Output information about the } \\
\text { point, function, and Jacobian at } \\
\text { the initial point. }\end{array}$ \\
\hline output_initial_scaling_statistics & yes & $\begin{array}{l}\text { Display matrix norms on the Ja- } \\
\text { cobian at the initial point. }\end{array}$ \\
\hline output_initial_statistics & no & $\begin{array}{l}\text { Output evaluatation of available } \\
\text { merit functions at the initial } \\
\text { point. }\end{array}$ \\
\hline output_model_statistics & yes & $\begin{array}{l}\text { Turns on or off printing of all the } \\
\text { statistics generated about the } \\
\text { model. }\end{array}$ \\
\hline return_best_point & yes & $\begin{array}{l}\text { Return the best point encoun- } \\
\text { tered or the absolute last iterate. }\end{array}$ \\
\hline
\end{tabular}

Table 1: Options added to PATH 4.0 


\section{Ill-Defined Models}

A problem can be ill-defined for several different reasons. We concentrate on the following particular cases. We will call $F$ well-defined at $\bar{x} \in C$ if $\bar{x} \in D$ and illdefined at $\bar{x}$ otherwise. Furthermore, we define $F$ to be well-defined near $\bar{x} \in C$ if there exists an open neighbourhood of $\bar{x}, \mathcal{N}(\bar{x})$, such that $C \cap \mathcal{N}(\bar{x}) \subseteq D$. By saying the function is well-defined near $\bar{x}$, we are simply stating that $F$ is defined for all $x \in C$ sufficiently close to $\bar{x}$. A function not well-defined near $\bar{x}$ is termed ill-defined near $\bar{x}$.

We will say that $F$ has a well-defined Jacobian at $\bar{x} \in C$ if there exists an open neighbourhood of $\bar{x}, \mathcal{N}(\bar{x})$, such that $\mathcal{N}(\bar{x}) \subseteq D$ and $F$ is continuously differentiable on $\mathcal{N}(\bar{x})$. Otherwise the function has an ill-defined Jacobian at $\bar{x}$. We note that a well-defined Jacobian at $\bar{x}$ implies that the MCP has a well-defined function near $\bar{x}$, but the converse is not true.

PATH uses both function and Jacobian information in its attempt to solve the MCP. Therefore, both of these definitions are relevant. We discuss cases where the function and Jacobian are ill-defined in the next two subsections. We illustrate uses for the merit function information and final point statistics within the context of these problems.

\subsection{Function Undefined}

We begin with a one-dimensional problem for which $F$ is ill-defined at $x=0$ as follows:

$$
0 \leq x \quad \perp \quad \frac{1}{x} \geq 0 .
$$

Here $x$ must be strictly positive because $\frac{1}{x}$ is undefined at $x=0$. This condition implies that $F(x)$ must be equal to zero. Since $F(x)$ is strictly positive for all $x$ strictly positive, this problem has no solution.

We are able to perform this analysis because the dimension of the problem is small. Preprocessing linear problems can be done by the solver in an attempt to detect obviously inconsistent problems, reduce problem size, and identify active components at the solution. Similar processing can be done for nonlinear models, but the analysis becomes more difficult to perform. Currently, PATH only checks the consistency of the bounds and removes fixed variables and the corresponding complementary equations from the model.

A modeler might not know a priori that a problem has no solution and might attempt to formulate and solve it. GAMS code for this model is provided in Figure 1. We must specify an initial value for $x$ in the code. If we were to not provide one, GAMS would use $x=0$ as the default value, notice that $F$ is undefined at the initial point, and terminate before giving the problem to PATH. The error message problem indicates that the function $\frac{1}{x}$ is ill-defined at $x=0$, but does not determine whether the corresponding MCP problem has a solution.

After setting the starting point, GAMS generates the model, and PATH proceeds to "solve" it. A portion of the output relating statistics about the 


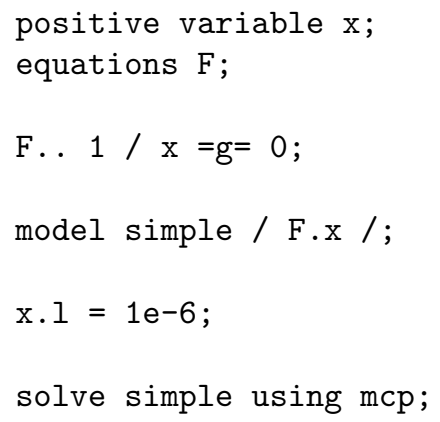

Figure 1: GAMS Code for Ill-Defined Function

FINAL STATISTICS

Inf-Norm of Complementarity . 1.0000e+00 eqn: (F)

Inf-Norm of Normal Map. . . . 1.1181e+16 eqn: (F)

Inf-Norm of Minimum Map . . . 8.9441e-17 eqn: (F)

Inf-Norm of Fischer Function. . 8.9441e-17 eqn: (F)

Inf-Norm of Grad Fischer Fcn. . 8.9441e-17 eqn: (F)

FINAL POINT STATISTICS

Maximum of X. . . . . . . 8.9441e-17 var: (X)

Maximum of F. . . . . . . . . 1.1181e+16 eqn: (F)

Maximum of Grad F. . . . . 1.2501e+32 eqn: (F)

var: (X)

Figure 2: PATH Output for Ill-Defined Function 
Figure 3: Merit Function Plot

solution is given in Figure 2. PATH uses the Fischer Function indicator as its termination criteria by default, but evaluates all of the merit functions given in Section 2 at the final point. The Normal Map merit function, and to a lesser extent, the complementarity error, indicate that the "solution" found does not necessarily solve the MCP.

To indicate the difference between the merit functions, Figure 3 plots them all for the simple example. We note that as $x$ approaches positive infinity, numerically, we are at a solution to the problem with respect to all of the merit functions except for the complementarity error, which remains equal to one. As $x$ approaches zero, the merit functions diverge, also indicating that $x=0$ is not a solution.

The natural residual and Fischer function tend toward 0 as $x \downarrow 0$. From these measures, we might think $x=0$ is the solution. However, as previously remarked $F$ is ill-defined at $x=0 . F$ and $\nabla F$ become very large, indicating that the function (and Jacobian) might not be well-defined. We might be tempted to conclude that if one of the merit function indicators is not close to zero, then we have not found a solution. This conclusion is not always the case. When one of the indicators is non-zero, we have reservations about the solution, but we cannot eliminate the possibility that we are actually close to a solution. If we slightly perturb the original problem to

$$
0 \leq x \quad \perp \quad \frac{1}{x+\epsilon} \geq 0
$$

for a fixed $\epsilon>0$, the function is well-defined over $C=\mathbf{R}_{+}^{n}$ and has a unique solution at $x=0$. In this case, by starting at $x>0$ and sufficiently small, all of the merit functions, with the exception of the Normal Map, indicate that we have solved the problem as is shown by the output in Figure 4 for $\epsilon=1 * 10^{-6}$ 
FINAL STATISTICS

Inf-Norm of Complementarity . . $1.0000 \mathrm{e}-14$ eqn: (G)
Inf-Norm of Normal Map. . . . $1.0000 \mathrm{e}+06$ eqn: (G)
Inf-Norm of Minimum Map . . . $1.0000 \mathrm{e}-20$ eqn: (G)
Inf-Norm of Fischer Function. . $1.0000 \mathrm{e}-20$ eqn: (G)
Inf-Norm of Grad Fischer Fcn. . $1.0000 \mathrm{e}-20$ eqn: (G)
FINAL POINT STATISTICS
Maximum of X. . . . . . . . . $1.0000 \mathrm{e}-20$ var: (X)
Maximum of F. . . . . . . . . $1.0000 \mathrm{e}+06$ eqn: (G)
Maximum of Grad F. . . . . . . $1.0000 \mathrm{e}+12$ eqn: (G)

Figure 4: PATH Output for Well-Defined Function

and $x=1 * 10^{-20}$. In this case, the Normal Map is quite large and we might think that the function and Jacobian are undefined. When only the normal map is non-zero, we may have just mis-identified the optimal basis. By setting the merit_function normal option, we can resolve the problem, identify the correct basis, and solve the problem with all indicators being close to zero. This example illustrates the point that all of these tests are not infallible. The modeler still needs to do some detective work to determine if they have found a solution or if the algorithm is converging to a point where the function is ill-defined.

\subsection{Jacobian Undefined}

Since PATH uses a Newton-like method to solve the problem, it also needs the Jacobian of $F$ to be well-defined. One model for which the function is well-defined over $C$, but for which the Jacobian is undefined at the solution is: $0 \leq x \perp-\sqrt{x} \geq 0$. This model has a unique solution at $x=0$.

Using PATH and starting from the point $x=1 * 10^{-14}$, PATH generates the output given in Figure 5. We can see the that gradient of the Fischer Function is nonzero and the Jacobian is beginning to become large. These conditions indicate that the Jacobian is undefined at the solution.

If we start from $x=0$, PATH correctly informs us that we are at the solution. Even though the entries in the Jacobian are undefined at this point, the GAMS interpreter incorrectly returns a value of 0 to PATH. This problem with the Jacobian is therefore undetectable by PATH.

When we are close to a point where $F$ is not differentiable, the Jacobian tends to have very large elements. This condition makes it hard to reliably calculate the Newton direction because at that point the Jacobian is likely poorly conditioned and can cause numerical problems with the linear algebra. It is therefore important for a modeler to inspect the given output to guard against such problems. 
FINAL STATISTICS

Inf-Norm of Complementarity . 1.0000e-07 eqn: (F)

Inf-Norm of Normal Map. . . . 1.0000e-07 eqn: (F)

Inf-Norm of Minimum Map . . . 1.0000e-07 eqn: (F)

Inf-Norm of Fischer Function. . 2.0000e-07 eqn: (F)

Inf-Norm of Grad FB Function. . 2.0000e+00 eqn: (F)

FINAL POINT STATISTICS

Maximum of X. . . . . . . 1.0000e-14 var: (X)

Maximum of F. . . . . . . . $1.0000 \mathrm{e}-07$ eqn: (F)

Maximum of Grad F..... 5.0000e+06 eqn: (F)

var: (X)

Figure 5: PATH Output for Ill-Defined Jacobian

\section{Poorly Scaled Models}

Problems which are well-defined can have various numerical problems that can impede the algorithm's convergence. One particular problem is a badly scaled Jacobian. In such cases, we can obtain a poor "Newton" direction because of numerical problems introduced in the linear algebra performed. This problem can also lead the code to a point from which it cannot recover.

The final model given to the solver should be scaled such that we avoid numerical difficulties in the linear algebra. The output provided by PATH can be used to iteratively refine the model so that we eventually end up with a well-scaled problem. We note that we only calculate our scaling statistics at the starting point provided. For nonlinear problems these statistics may not be indicative of the overall scaling of the model. Model specific knowledge is very important when we have a nonlinear problem because it can be used to appropriately scale the model to achieve a desired result.

We look at a contact problem, the kyh.gms model in MCPLIB, that has some scaling problems. The relevant output from PATH for the original code is given in Figure 6. The maximum row norm is defined as

$$
\max _{1 \leq i \leq n} \sum_{1 \leq j \leq n}\left|(\nabla F(x))_{i j}\right|
$$

and the minimum row norm is

$$
\min _{1 \leq i \leq n} \sum_{1 \leq j \leq n}\left|(\nabla F(x))_{i j}\right|
$$

Similar definitions are used for the column norm. The norm numbers for this particular example are not extremely large, but we can nevertheless improve the scaling. We first decided to reduce the magnitude of the DN block of equations as indicated by PATH. Using the GAMS modeling language, we can scale particular 
INITIAL POINT STATISTICS

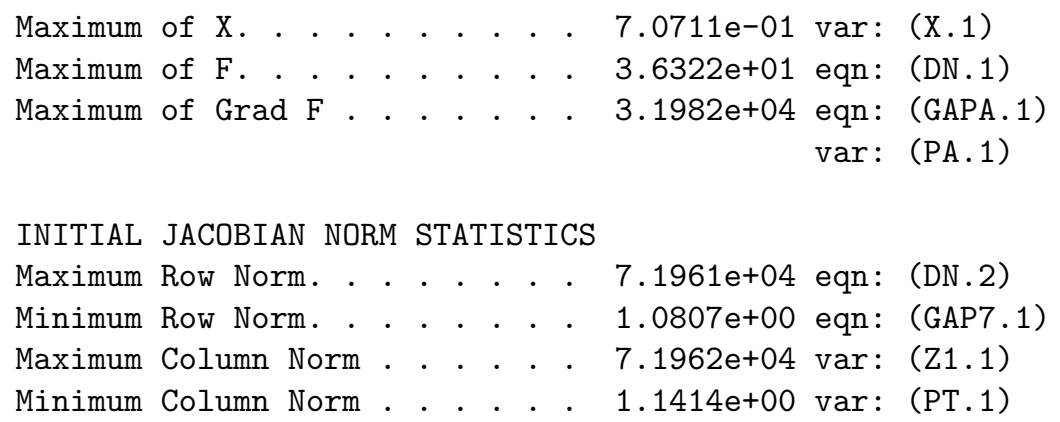

Figure 6: PATH Output - Poorly Scaled Model

INITIAL POINT STATISTICS

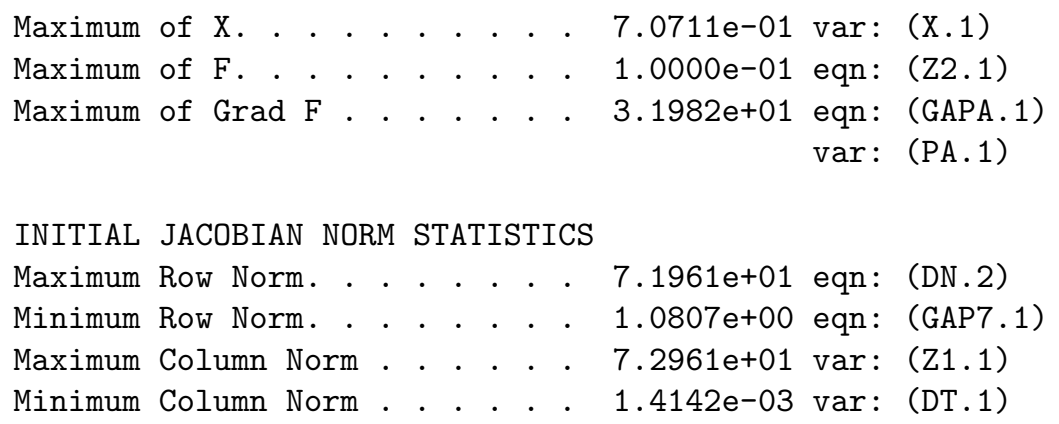

Figure 7: PATH Output - Well-Scaled Model

equations and variables using the scale attribute. To turn the scaling on for the model we use the .scaleopt model attribute. After scaling the DN block, we re-ran PATH and found two additional blocks of equations that also needed scaling, GAPA and GAPB. The code added to the model follows:

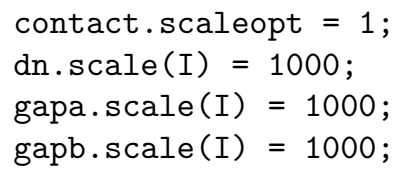

The modified model has been called kyh-scale.gms. After scaling these blocks of equations in the model, we have improved the scaling statistics which are given in Figure 7 for the new model. Using the scaling language features and the information provided by PATH we are able to remove some of the problem's difficulty and obtain better performance from PATH. For this particular problem PATH had difficulty solving the unscaled model, but was able to easily solve the correctly scaled problem. It is possible to get even more information on initial 
INITIAL POINT STATISTICS

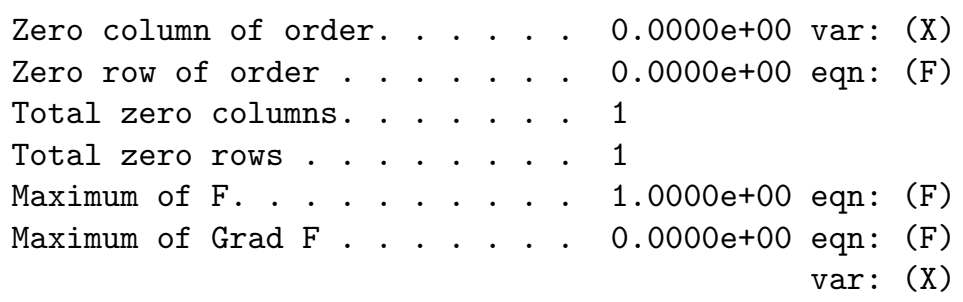

Figure 8: PATH Output - Zero Rows and Columns

point scaling by inspecting the GAMS listing file. The equation row listing gives the values of all the entries of the Jacobian at the starting point. The row norms generated by PATH give good pointers into this source of information.

Not all of the numerical problems are directly attributable to poorly scaled models. Problems for which the Jacobian of the active constraints is singular or nearly singular can also cause numerical difficulty as illustrated next.

\section{$5 \quad$ Singular Models}

Assuming that the problem is well-defined and properly scaled, we can still have a Jacobian for which the active constraints are singular or nearly singular (i.e. it is ill-conditioned). When problems are singular or nearly singular, we are also likely to have numerical problems. As a result the "Newton" direction obtained from the linear problem solver can be very bad. In PATH, we can use proximal perturbation or add artificial variables to attempt to remove the singularity problems from the model. However, it is most often beneficial for solver robustness to remove singularities if possible.

The easiest problems to detect are those for which the Jacobian has zero rows and columns. A simple problem for which we have zero rows and columns is:

$$
-2 \leq x \leq 2 \quad \perp \quad-x^{2}+1 .
$$

Note that the Jacobian, $-2 x$, is non-singular at all three solutions, but singular at the point $x=0$. Output from PATH on this model starting at $x=0$ is given in Figure 8. We display in the code the variables and equations for which the row/column in the Jacobian is close to zero. These situations are problematic and for nonlinear problems likely stem from the modeler providing an inappropriate starting point or fixing some variables resulting in some equations becoming constant. We note that the solver may perform well in the presence of zero rows and/or columns, but the modeler should make sure that these are what was intended.

Singularities in the model can also be detected by the linear solver. This in itself is a hard problem and prone to error. For matrices which are poorly 
scaled, we can incorrectly identify "linearly dependent" rows because of numerical problems. Setting output_factorization_singularities yes in an options file will inform the user which equations the linear solver thinks are linearly dependent. Typically, singularity does not cause a lot of problems and the algorithm can handle the situation appropriately. However, an excessive number of singularities are cause for concern. A further indication of possible singularities at the solution is the lack of quadratic convergence to the solution.

We now turn our attention towards using the diagnostic information provided by PATH to improve an actual model.

\section{Von Thunen Land Model}

In this section, we describe the Von Thunen land model, a problem renowned in the mathematical programming literature for its computational difficulty. We attempt to understand more carefully the facets of the problem that make it difficult to solve. This will enable us in the sequel to outline and identify these problems and furthermore to extend the model to a more realistic and computationally more tractable form.

\subsection{Classical Model}

The problem is cast in the Arrow-Debreu framework as an equilibrium problem. The basic model is a closed economy consisting of three economic agents, a landowner, a worker and a porter. There is a central market, around which concentric regions of land are located. Since the produced goods have to be delivered to the market, this is an example of a spatial price equilibrium. The key variables of the model are the prices of commodities, land, labour and transport. Given these prices, it is assumed that the agents demand certain amounts of the commodities, which are supplied so as to maximize profit in each sector. Walras' law is then a consequence of the assumed competitive paradym, namely that supply will equal demand in the equilibrium state.

We now describe the problems that the consumers and the producers face. We first look at consumption, and derive a demand function for each of the consumer agents in the economy. Each of these agents has a utility function, that they wish to maximize subject to their budgetary constraints. As is typical in such problems, the utility function is assumed to be Cobb-Douglas

$$
u_{a}(d)=\prod_{c} d_{c}^{\alpha_{c, a}}, \quad \alpha_{c, a} \geq 0, \sum_{c} \alpha_{c, a}=1,
$$

where the $\alpha_{c, a}$ are given parameters dependent only on the agent. For each agent $a$, the variables $d_{c}$ represent quantities of the desired commodities $c$. In the Von Thunen model, the goods are wheat, rice, corn and barley. The agents endowments determine their budgetary constraint as follows. Given current market prices, an agents wealth is the value of the initial endowment of goods 
at those prices. The agents problem is therefore

$$
\max _{d} u_{a}(d) \text { subject to }\langle p, d\rangle \leq\left\langle p, e_{a}\right\rangle, d \geq 0
$$

where $e_{a}$ is the endowment bundle for agent a. A closed form solution, corresponding to demand from agent $a$ for commodity $c$ is thus

$$
d_{c, a}(p):=\frac{\alpha_{c, a}\left\langle p, e_{a}\right\rangle}{p_{c}} .
$$

Note that this assumes the prices of the commodities $p_{c}$ are positive.

The supply side of the economy is similar. The worker earns a wage $w_{L}$ for his labour input. The land is distributed around the market in rings with a rental rate $w_{r}$ associated with each ring $r$ of land. The area of land $a_{r}$ in each ring is an increasing function of $r$. The model assumes that labour and land are substitutable via a constant elasticities of substitution (CES) function.

Consider the production $x_{c, r}$ of commodity $c$ in region $r$. In order to maximize profit (or minimize costs), the labour $y_{L}$ and land use $y_{r}$ solve

$$
\min w_{L} y_{L}+w_{r} y_{r} \text { subject to } \phi_{c} y_{L}^{\beta_{c}} y_{r}^{1-\beta_{c}} \geq x_{c, r}, y_{L}, y_{r} \geq 0
$$

where $\phi_{c}$ is a given cost function scale parameter. The technology constraint is precisely the CES function allowing a suitable mix of labour and land use. Again, a closed form solution can be calculated. For example, the demand for labour in order to produce $x_{c, r}$ of commodity $c$ in region $r$ is given by

$$
x_{c, r} \frac{\beta_{c}\left(\frac{w_{L}}{\beta_{c}}\right)^{\beta_{c}}\left(\frac{w_{r}}{1-\beta_{c}}\right)^{1-\beta_{c}}}{\phi_{c} w_{L}} .
$$

Considering all such demands, this clearly assumes the prices of inputs $w_{L}, w_{r}$ are positive. A key point to note is that input commodity (factor) demands to produce $x_{c, r}$ can be determined by first solving (1) for unit demand $x_{c, r} \equiv 1$ and then multiplying these factor demands by the actual amount desired. Using this fact, the unit production cost $\gamma_{c, r}$ for commodity $c$ in region $r$ can be calculated as follows:

$$
\begin{aligned}
\gamma_{c, r} & =w_{L} \bar{y}_{L}+w_{r} \bar{y}_{r} \\
& =w_{L} \frac{\beta_{c}\left(\frac{w_{L}}{\beta_{c}}\right)^{\beta_{c}}\left(\frac{w_{r}}{1-\beta_{c}}\right)^{1-\beta_{c}}}{\phi_{c} w_{L}}+w_{r} \frac{\left(1-\beta_{c}\right)\left(\frac{w_{L}}{\beta_{c}}\right)^{\beta_{c}}\left(\frac{w_{r}}{1-\beta_{c}}\right)^{1-\beta_{c}}}{\phi_{c} w_{r}} \\
& =\frac{1}{\phi_{c}}\left(\frac{w_{L}}{\beta_{c}}\right)^{\beta_{c}}\left(\frac{w_{r}}{1-\beta_{c}}\right)^{1-\beta_{c}} .
\end{aligned}
$$

Transportation is provided by a porter, earning a wage $w_{p}$. If we denote the unit cost for transportation of commodity $c$ by $t_{c}$, then unit transportation cost to market is

$$
T_{c, r}\left(w_{p}\right):=t_{c} d_{r} w_{p}
$$


where $d_{r}$ is the distance of region $r$ to the market. Spatial price equilibrium arises from the consideration:

$$
0 \leq x_{c, r} \perp \gamma_{c, r}\left(w_{L}, w_{r}\right)+T_{c, r}\left(w_{p}\right) \geq p_{c} .
$$

This is intuitively clear; it states that commodity $c$ will be produced in region $r$ only if the combined cost of production and transportation equals the market price.

The above derivations assumed that the producers and consumers acted as price takers. Walras' law is now invoked to determine the prices so that markets clear. The resulting complementarity problem is:

$$
\begin{gathered}
\gamma_{c, r}=\frac{1}{\phi_{c}}\left(\frac{w_{L}}{\beta_{c}}\right)^{\beta_{c}}\left(\frac{w_{r}}{1-\beta_{c}}\right)^{1-\beta_{c}} \\
0 \leq x_{c, r} \quad \perp \quad \gamma_{c, r}+T_{c, r}\left(w_{P}\right) \geq p_{c} \\
0 \leq w_{L} \quad \perp \quad e_{L} \geq \sum_{r, c} x_{c, r} \frac{\beta_{c} \gamma_{c, r}}{w_{L}} \\
0 \leq w_{r} \quad \perp \quad a_{r} \geq \sum_{c} \frac{x_{c, r}\left(1-\beta_{c}\right) \gamma_{c, r}}{w_{r}} \\
0 \leq w_{P} \quad \perp \quad e_{P} \geq \sum_{r, c} t_{c} d_{r} x_{c, r} \\
0 \leq p_{c} \quad \perp \quad \sum_{r} x_{c, r} \geq \frac{\alpha_{c, P} e_{P} w_{P}+\alpha_{c, L} e_{L} w_{L}+\alpha_{c, O} \sum_{r} w_{r} a_{r}}{p_{c}}
\end{gathered}
$$

Note that in (4), (5) and (6), the amounts of labour, land and transport are bounded from above, and hence the prices on these inputs are determined as multipliers (or shadow prices) on the corresponding constraints. The final relationship (7) in the above complementarity problem corresponds to market clearance; prices are nonnegative and can only be positive if supply equals demand. (Some modelers multiply the last inequality throughout by $p_{c}$. This removes problems where $p_{c}$ becomes zero, but can also introduce spurious solutions.)

The Arrow-Debreu theory guarantees that the problem is homogeneous in prices; $(x, \lambda w, \lambda p)$ is also a solution whenever $(x, w, p)$ solves the above. Typically this singularity in the model is removed by fixing a numeraire, that is fixing a price (for example $w_{L}=1$ ) and dropping the corresponding complementary relationship.

Unfortunately, in this formulation even after fixing a numeraire, some of the variables $p$ and $w$ may go to zero, resulting in an ill-defined problem. In the case of the Von Thunen land model, the rental price of land $w_{r}$ decreases as the distance to market increases, and for remote rings of land, it becomes zero. A standard modeling fix is to put artificial lower bounds on these variables. Even with this fix, the problem typically remains very hard to solve. More importantly, the homogeneity property of the prices used above to fix a numeraire no longer holds, and the corresponding complementary relationship (which was 
dropped from the problem) may fail to be satisfied. It therefore matters which numeriare is fixed, and many modelers run into difficulty since in many cases the solution found by a solver is invalid for the originally posed model.

In order to test our diagnostic information, we implemented a version of the above model in GAMS. The model corresponds closely to the MCPLIB model pgvon105.gms except we added more regions to make the problem even more difficult. The model file has been documented more fully, and the data rounded to improve clarity.

The first trial we attempted was to solve the model without fixing a numeraire. In this case, PATH 4.0 failed to find a solution. At the starting point, the indicators described in Section 3 are reasonable, and there are no zero rows/columns in the Jacobian. At the best point found, all indicators are still reasonable. However, the listing file indicates a lare number of division by zero problems occurring in (5). We also note that a nonzero proximal perturbation is used in the first iteration of the crash method. This is an indication of singularities. We therefore added an option to output factorization singularities, and singularities appeared in the first iteration. At this point, we decided to fix a numeraire to see if this alleviated the problem.

We chose to fix the labour wage rate to 1 . After increasing the iterations allowed to 100,000, PATH 4.0 solved the problem. The statistics at the solution are cause for concern. In particular, the gradient of the Fischer function is 7 orders of magnitude larger than all the other residuals. Furthermore, the Jacobian is very large at the solution point. Looking further in the listing file, a large number of division by zero problems occur in (5).

To track down the problem further, we added an artifical lower bound on the variables $w_{r}$ of $10^{-5}$, that would not be active at the aforementioned solution. Resolving gave the same "solution", but resulted in the domain errors disappearing.

Although the problem is solved, there is concern on two fronts. Firstly, the gradient of the Fischer function should go to zero at the solution. Secondly, if a modeler happens to make the artificial lower bounds on the variables a bit larger, then they become active at the solution, and hence the constraint that has been dropped by fixing the price of labour at 1 is violated at this point. Of course, the algorithm is unable to detect this problem, since it is not part of the model that is passed to it, and the corresponding output looks satisfactory.

We are therefore led to the conclusion that the model as posulated is illdefined. The remainder of this section outlines two possible modeling techniques to overcome the difficulties with ill-defined problems of this type.

\subsection{Intervention Pricing}

The principal difficulty is the fact that the rental prices on land go to zero as proximity to the market decreases, and become zero for sufficiently remote rings. Such a property is unlikely to hold in a practical setting. Typically, a landowner has a minimum rental price (for example, land in fallow increases in value). As outlined above, a fixed lower bound on the rental price violates 
the well-establised homogeneity property. A suggestion postulated by Professor Thomas Rutherford is to allow the landowner to intervene and "purchase-back" his land whenever the rental cost gets smaller than a certain fraction of the labour wage.

The new model adds a (homogeneous in price) constraint

$$
0 \leq i_{r} \quad \perp \quad w_{r} \geq 0.0001 * w_{L}
$$

and modifies (5) and (7) as follows:

$$
\begin{aligned}
& 0 \leq w_{r} \quad \perp \quad a_{r}-i_{r} \geq \sum_{c} \frac{x_{c, r}\left(1-\beta_{c}\right) \gamma_{c, r}}{w_{r}} \\
& 0 \leq p_{c} \quad \perp \quad \sum_{r} x_{c, r} \geq \frac{\alpha_{c, P} e_{P} w_{P}+\alpha_{c, L} e_{L} w_{L}+\alpha_{c, O} \sum_{r} w_{r}\left(a_{r}-i_{r}\right)}{p_{c}} .
\end{aligned}
$$

Given the intervention purchase, we can now add a lower bound on $w_{r}$ to avoid division by zero errors. In our model we chose $10^{-5}$ since this will never be active at the solution and therefore will not affect the positive homogeneity. After this reformulation, PATH 4.0 solves the problem. Furthermore, the gradient of the Fischer function, although slightly larger than the other residuals, is quite small, and can be made even smaller by reducing the convergence tolerance of PATH. Inspecting the listing file, the only difficulties mentioned are division by zero errors in the market clearance condition (8), that can be avoided a posteori by imposing an artificial (inactive) lower bound on these prices. We chose not to do this however.

\subsection{Nested Production and Maintenance}

Another observation that can be used to overcome the land price going to zero is the fact that land typically requires some maintenance labour input to keep it usable for crop growth. Traditionally, in economics, this is carried out by providing a nested CES function as technology input to the model. The idea is that commodity $c$ in region $r$ is made from labour and an intermediate good. The intermediate good is "maintained land". Essentially, the following production problem replaces (1):

$$
\begin{array}{cc}
\min _{y_{M}, y_{L}, y_{r}, g} & w_{L}\left(y_{M}+y_{L}\right)+w_{r} y_{r} \\
\text { subject to } & y_{r} \geq\left(1-\beta_{c}-\epsilon\right) g \\
& y_{M} \geq \epsilon g \\
\phi_{c} y_{c}^{\beta_{c}} g^{1-\beta_{c}} \geq 1 \\
y_{M}, y_{L}, y_{r}, g \geq 0 .
\end{array}
$$

Note that the variable $y_{M}$ represents "maintenance labour" and $g$ represents the amount of "maintained land" produced, an intermediate good. The process of generating maintained land uses a Leontieff production function, namely

$$
\min \left(\lambda_{r} y_{r}, \lambda_{M} y_{M}\right) \geq g
$$


Here $\lambda_{M}=\frac{1}{\epsilon}, \epsilon$ small, corresponds to small amounts of maintenance labour, while $\lambda_{r}=\frac{1}{1-\beta_{c}-\epsilon}$ is chosen to calibrate the model correctly. A simple calculus exercise then generates appropriate demand and cost expressions. The resulting complementarity problem comprises (3), (6), (7) and

$$
\begin{gathered}
\gamma_{c, r}=\frac{w_{L}^{\beta_{c}}}{\phi_{c}}\left(\frac{w_{L} \epsilon+w_{r}\left(1-\beta_{c}-\epsilon\right)}{1-\beta_{c}}\right)^{1-\beta_{c}} \\
0 \leq w_{L} \quad \perp \quad e_{L} \geq \sum_{r, c} x_{c, r} \gamma_{c, r}\left(\frac{\beta_{c}}{w_{L}}+\frac{\epsilon\left(1-\beta_{c}\right)}{w_{L} \epsilon+w_{r}\left(1-\beta_{c}-\epsilon\right)}\right) \\
0 \leq w_{r} \quad \perp \quad a_{r} \geq \sum_{c} \frac{x_{c, r} \gamma_{c, r}\left(1-\beta_{c}\right)\left(1-\beta_{c}-\epsilon\right)}{w_{L} \epsilon+w_{r}\left(1-\beta_{c}-\epsilon\right)}
\end{gathered}
$$

After making the appropriate modifications to the model file, PATH 4.0 solved the problem on defaults without any difficulties. All indicators showed he problem and solution found to be well-posed.

\section{Conclusion}

Models which are ill-defined, poorly scaled, or singular can cause algorithms to have extreme difficulty. Identifying the problem is a non-trivial matter for the modeler. An algorithm can provide additional statistics about the model to the modeler. This information can help the modeler to quickly identify and correct the problem.

Further algorithmic work would be useful; in particular methods to automatically identify implied lower bounds may help to avoid many of the domain violations that occur in ill-defined models.

\section{Acknowledgement}

The authors wish to thank Thomas Rutherford for numerous enlightening discussions of the von Thunen model and suggestions for improvements to the output of PATH that are relevant to modelers.

\section{References}

[1] A. Brooke, D. Kendrick, and A. Meeraus. GAMS: A User's Guide. The Scientific Press, South San Francisco, CA, 1988.

[2] S. P. Dirkse and M. C. Ferris. MCPLIB: A collection of nonlinear mixed complementarity problems. Optimization Methods and Software, 5:319-345, 1995.

[3] S. P. Dirkse and M. C. Ferris. The PATH solver: A non-monotone stabilization scheme for mixed complementarity problems. Optimization Methods and Software, 5:123-156, 1995. 
[4] S. P. Dirkse and M. C. Ferris. A pathsearch damped Newton method for computing general equilibria. Annals of Operations Research, 1996.

[5] S. P. Dirkse and M. C. Ferris. Crash techniques for large-scale complementarity problems. In Ferris and Pang [11], pages 40-61.

[6] M. C. Ferris, R. Fourer, and D. M. Gay. Expressing complementarity problems and communicating them to solvers. Mathematical Programming Technical Report 98-02, Computer Sciences Department, University of Wisconsin, Madison, Wisconsin, 1998.

[7] M. C. Ferris, C. Kanzow, and T. S. Munson. Feasible descent algorithms for mixed complementarity problems. Mathematical Programming Technical Report 98-04, Computer Sciences Department, University of Wisconsin, Madison, Wisconsin, 1998.

[8] M. C. Ferris and S. Lucidi. Nonmonotone stabilization methods for nonlinear equations. Journal of Optimization Theory and Applications, 81:53-71, 1994.

[9] M. C. Ferris and T. S. Munson. Complementarity problems in GAMS and the PATH solver. Mathematical Programming Technical Report 98-12, Computer Sciences Department, University of Wisconsin, Madison, Wisconsin, 1998.

[10] M. C. Ferris and T. S. Munson. Interfaces to PATH 3.0: Design, implementation and usage. Computational Optimization and Applications, forthcoming, 1998.

[11] M. C. Ferris and J. S. Pang, editors. Complementarity and Variational Problems: State of the Art, Philadelphia, Pennsylvania, 1997. SIAM Publications.

[12] M. C. Ferris and J. S. Pang. Engineering and economic applications of complementarity problems. SIAM Review, 39:669-713, 1997.

[13] R. Fourer, D.M. Gay, and B.W. Kernighan. AMPL: A Modeling Language for Mathematical Programming. Duxbury Press, 1993.

[14] T. F. Rutherford. Applied general equilibrium modeling with MPSGE as a GAMS subsystem: An overview of the modeling framework and syntax. Computational Economics, forthcoming, 1998.

[15] P. Tseng. Growth behavior of a class of merit functions for the nonlinear complementarity problem. Journal of Optimization Theory and Applications, 89:17-37, 1996. 